\title{
Los Mapas Contables de Gestión de las Empresas Cotizadas Chilenas: Análisis Cualitativo
}

\author{
Miguel A. Pérez-Benedito, Luis Porcuna-Enguix y Rubén Porcuna-Enguix \\ Facultad de Economía, Departamento de Contabilidad, Universidad de Valencia, Avenida dels Tarongers \\ s/n 46022 Valencia. -España (e-mail: miguel.a.perez@uv.es, luis.porcuna@uv.es, ruben.porcuna@uv.es)
}

Recibido May. 20, 2016; Aceptado Jul. 13, 2016; Versión final Sep. 30, 2016, Publicado Feb. 2017

\begin{abstract}
Resumen
El artículo analiza las clases de gestión realizadas por las empresas cotizadas chilenas localizadas en los espacios administrativos de Chile. La orientación del análisis realizado considera que el factor cultural de la región donde están localizadas las empresas chilenas condiciona la clase de gestión desarrollada por dichas empresas. El desarrollo de esta hipótesis se realiza aplicando la metodología contable de los gráficos radiales. Las tensiones existentes entre los periodos medios de maduración representados en un gráfico radial asumen la existencia de un factor cultural en las transacciones realizadas por las empresas en una región y supone que la toma de decisión en un área tiene efectos multidireccionales. De esta forma, las decisiones de economía general sobre un sector, así como el contraste de la actividad empresarial respecto a los valores medios de los indicadores del sector, son cuestionables.
\end{abstract}

Palabras clave: mapas de gestión, metodología contable, factor cultural

\section{The Accounting Maps of Management of Chilean Listed Companies: Qualitative Analysis}

\begin{abstract}
The paper analyzes the kinds of management performed by Chilean listed companies located in geographical government spaces in Chile. The guide of the analysis done in this work considers the cultural factor of the region where Chilean companies are located affects the kind of management done by companies. The development of this hypothesis is made applying the accounting methodology of radar charts. The tensions existing between average period of maturation, which are represented on a radar chart, assumes that there exists a cultural factor on transactions made by companies in a region, and that the making-decision process on a management area has multidimensional effects. On this way, the decisions on general economy in a sector as well as the contrast of business activities respect to the mean values of a sector indicator are questionable.
\end{abstract}




\section{INTRODUCCION}

El análisis de la gestión de las empresas presenta un campo de estudio de amplio alcance donde el objetivo último perseguido en la investigación determina la metodología óptima que debe ser aplicada. Sin embargo, adoptar respuestas óptimas para superar oportunamente las perturbaciones actuales de los mercados son acciones complejas ante la ausencia de sucesos pasados con idénticas características. La respuesta generalizada de los organismos reguladores de los mercados ante las crisis financieras ha sido obtener indicadores más consistentes para evaluar la acción de las instituciones financieras y no financieras, dirigidos a facilitar la toma de decisiones a todas las partes interesadas en su actividad, con objeto de evitar trasladar su riesgo moral sobre el conjunto de la economía. En este sentido, la metodología contable de los gráficos radiales considera que las decisiones empresariales afectan al conjunto de las actividades que realizan las empresas y analiza sus efectos en sentido multidireccional. La incorporación de los periodos medios de maduración en los ejes de un gráfico radial permite observar las consecuencias de las decisiones empresariales en tantas dimensiones como áreas de gestión estén representadas en el mismo, Las distancias obtenidas entre los periodos medios de maduración, representan las tensiones existentes en cada una de las áreas de la actividad empresarial representadas en un gráfico radial. La mayor o menor distancia entre los periodos medios de maduración está asociada a variables macroeconómicas de las regiones donde las empresas se encuentran localizadas y vinculadas con la forma de realizar las transacciones, incorporando de manera indirecta, los factores culturales de las regiones a la actividad empresarial. (Martínez y Pérez, 2011; Pérez, 2015)

El factor cultural en la teoría de la transacción de Williamson (Salgado, 2003; García y Taboada, 2012) o en la teoría de localización de las empresas de Krugman (Ascani et al, 2012), actúa como un componente subjetivo en ambas teorías. La influencia de este componente en la teoría institucional de North se ve reflejado al considerar la acción humana como el componente principal que actúa en la actividad empresarial (Cárdenas. y Ojeda, 2002; Urbano y Díaz, 2007). La existencia de este factor en el ámbito interno de las empresas ha permitido el desarrollo de distintas líneas de investigación (Espinosa y Salinas, 2013, 2015; Hernández et al, 2014). No obstante, la influencia de los entornos económicos sobre las transacciones realizadas por las entidades, tanto en el ámbito interno como externo de las mismas, pone de manifiesto la existencia de una cultura económica identificada con las clases de gestión desarrolladas en los mismos. Las clases de gestión, se identifican con las figuras geométricas que se generan al incorporar los periodos medios de maduración en un gráfico radial, obtenidos como resultantes de las transacciones realizadas. De esta forma, inversores, analistas, consultores o gestores de empresas obtienen una percepción multidimensional de la actividad empresarial con efectos patrimoniales que, posteriormente, pueden confirmar con sus propios indicadores. Por otra parte, la vinculación entre un gráfico radial o clase de gestión y la situación patrimonial de las empresas está garantizada siempre que los periodos medios de maduración se obtengan desde la información que facilitan los estados financieros. Esta relación ha determinado que, en el estudio desarrollado en este artículo, se elijan las empresas cotizadas chilenas, de acuerdo con la calidad de la información contable que deben presentar sus estados financieros, para analizar la economía chilena y justificar la existencia de mapas de gestión que identifican regiones económicas de acuerdo con las clases de gestión que adoptan las empresas localizadas en ellas.

El mapa de gestión está compuesto por el conjunto de empresas que adoptan una misma clase de gestión en un lugar geográfico y en un momento del tiempo. Mantener una clase de gestión durante un periodo de tiempo, indica la existencia de una cultura económica consolidada que no es alterada por las perturbaciones económicas o financieras o por las alteraciones en las condiciones técnicas de la región donde se encuentran localizadas las empresas, como modificaciones de los medios de comunicación social, terrestres, marítimos o aéreos o en la estructura del propio territorio. En consecuencia, el artículo limita su desarrollo al análisis cualitativo de la actividad de las empresas cotizadas chilenas. Se desarrolla bajo la hipótesis de que las empresas adoptan formas de gestión comunes en un territorio, independientemente del sector económico al que pertenecen. De acuerdo con lo indicado, el resto del artículo se estructura como sigue. En la segunda sección se explica aquellos factores culturas y su influencia en el éxito empresarial de las compañías. La sección tres consta del desarrollo teórico de la metodología contable de los gráficos radiales y su interpretación en la gestión empresarial. En la cuarta sección analizamos la visión práctica de los diferentes mapas de gestión adoptadas por las empresas chilenas durante el periodo comprendido entre 2005 y 2013, analizando el efecto de las crisis financieras en la economía chilena. En la quinta parte se visualiza la distribución geográfica de las diferentes clases de gestión empresarial y, por último, la sexta parte reúne las principales conclusiones de este estudio.

\section{FACTORES CULTURALES Y SU INFLUENCIA EN EL ÉXITO EMPRESARIAL}

El centro de la geografía económica y la economía internacional se basa en que los intercambios económicos son consecuencia, entre otros factores, de la distribución aleatoria de los recursos naturales en 
el espacio geográfico (Ricardo, 1817). Alcacer y Delgado (2016) exponen que la mayoría de investigación económica (p.ej. Jacobs, 1984; Krugman, 1991a; Delgado et al., 2010) y de estrategia empresarial (p.ej. Alcácer y Chung, 2014) se centra en elementos del entorno externo, en particular las economías de aglomeración (ya sea externa o interna), como factor influyente en la localización de las empresas. De hecho, Porter (1990) empezó a introducir el concepto de clusters y puso de manifiesto los motivos del éxito competitivo empresarial: (i) la concentración geográfica de agentes económicos especializados, y (ii) la existencia de factores determinantes de la competitividad territorial. En este sentido, varios trabajos han desarrollado nuevas metodologías o guías para realizar un análisis estratégico con el fin de detectar oportunidades en su entorno para establecer adecuadas estructuras organizativas a partir de la colaboración y la cooperación entre las empresas constituyentes del cluster (Capó et al., 2007b) o a partir de la correcta gestión del conocimiento (Capó et al., 2007a).

La literatura previa destaca dos tipos de distancias entre las empresas, las cuales favorecen o dificultan el desempeño empresarial: (i) la psíquica y la física. Por un lado, la distancia psíquica depende a su vez de la proximidad cultural e institucional entre el país de origen y de destino. Du et al. (2008) estudia las Empresas de Inversión Extranjera (EIE) y argumenta que las EIE dan prioridad a mercados percibidos como psicológicamente más cercanos. Las EIE cercanas institucionalmente puede aprovechar la proximidad de sus agencias gubernamentales y burocráticas para conseguir mayor eficiencia en sus operaciones empresariales, sin embargo, el posible impacto negativo de una mayor diferencia institucional puede aliviarse por la proximidad cultural. Por otra parte, de acuerdo a Gómez-Guillamón y Sanchez-Val (2012), podemos confirmar que la distancia física considera la existencia de dos efectos espaciales: (i) la localización, y (ii) un efecto indirecto o diferenciador. Por ejemplo, muchos trabajos destacan la importancia del factor geográfico para analizar los tipos de auditores y la calidad resultante de su trabajo (Hope et al., 2008; Wang et al., 2008; Huang y Li, 2009). El efecto de localización implica que aquellas provincias con un desarrollo económico e institucional mayor muestran valores de calidad de auditoría mayores, y que a su vez existe un efecto diferenciador positivo, es decir, que estos valores de calidad de auditoría superiores no dependen únicamente de las características propias de la provincia, sino también de la influencia de las zonas adyacentes (Krugman, 1991b).

Así pues, siguiendo la línea de Kalnins y Lafontaine (2013), la distancia es un factor que afecta al desempeño empresarial porque: (i) limita el flujo de bienes, capital, e información entre países y/o empresas localizadas en un mismo cluster (p.ej. Lerner, 1995; Adams y Jaffe 1996; Coval and Moskowitz 2001; Portes and Rey 2005), (ii) afecta a las decisiones de la forma de la organización (p.ej. Kalnins y Lafontaine, 2004; Lafontaine y Shaw, 2005), y (iii) afecta al comportamiento del consumidor (Bronnenberg et al., 2009). Por ende, la consecuencia inmediata de una mayor distancia es un menor comercio entre las empresas, puesto que representa una menor productividad, mayores costes de transporte y monitorización, y un menor flujo y transmisión de información entre el origen y destino. Autores como Rauch (2001), Anderson y van Wincoop (2004) y Allen (2014) definen una mayor distancia como una mayor fricción de información y una menor concentración geográfica supone, en principio, menores ventajas competitivas (Porter, 1996), repele el trabajo conjunto de grandes grupos empresariales y la asociación con proveedores especializados (Marshall, 1920). Estos efectos se encuentran presenten en el análisis de las clases de gestión existentes en Chile que han sido obtenidas a través de la metodología contables de los gráficos radiales.

\section{LA METODOLOGIA CONTABLE DE LOS GRAFICOS RADIALES}

La metodología contable de los gráficos contables evalúa la actividad de las empresas a través de la significación económica y financiera de las relaciones de los ángulos internos de los gráficos radiales generados al incorporar los periodos medios de maduración de la empresa en los ejes de un gráfico radial. Para obtener el valor de los coeficientes angulares se deben de aplicar la teoría del coseno y la teoría del seno de la geometría plana. A través de la aplicación de la teoría del coseno se obtienen las distancias perimetrales (DPk) o la longitud de los lados de la figura geométrica generada al unir linealmente los ejes de un gráfico radial. Una vez obtenida la distancia de los lados del perímetro de la figura geométrica generada se puede aplicar la teoría del seno y, de esta forma, obtener el valor de los ángulos internos de cada uno de los triángulos que forman un gráfico radial. Representada geométricamente una forma de gestión, los cambios en las partidas y contrapartidas que permiten obtener los periodos de maduración suponen cambios en la figura geométrica generada. Estos cambios afectan al valor de los ángulos internos de un gráfico radial en sentido multidimensional, de acuerdo con la naturaleza explicativa de los gráficos radiales. En consecuencia, en esta metodología, se observa como las decisiones adoptadas sobre un eje radial repercuten al mismo tiempo en las distintas áreas representadas en el mismo.

En este artículo, analizamos las clases de gestión adoptadas por las empresas chilenas y, como ejemplo ilustrativo, se representa en la Fig.1 las actuaciones de dos empresas localizadas en la región de Las Condes. En cada eje radial están representados los respectivos periodos de venta (pv), periodos de cobro 
(pc) y periodos de pago (pp). El efecto de la crisis financiera de Lehman Brothers en 2009 supone un cambio de gestión con repercusiones en el año 2013, cuando la crisis financiera de la Deuda Soberana está produciendo sus efectos. El cambio de gestión común para ambas sociedades se ha producido en el área de tesorería. El aumento de la dilatación de las distancias perimetrales en tesorería (DP2) en el año 2013, supone una menor tensión en la liquidación de las operaciones comerciales y son consecuencia de la situación de crisis financiera en ese año. La diferencia entre ambas compañías se basa en la clase de gestión. La empresa BSA dirige su gestión hacia el cliente, dado que la distancia perimetral DP1 es la mayor que la distancia DP3, y la empresa PVR dirige su gestión en la obtención de crédito de la cartera de proveedores, dado que la distancia perimetral DP3 es mayor que la distancia perimetral DP1. Las distintas clases de gestión tienen sus efectos en la estructura financiera de las empresas. La gestión dirigida al cliente supone ceder mayor nivel de crédito a los clientes que la obtenida de los proveedores y la gestión continuada de la empresa BSA estará supeditada a la liquidez de la cartera de clientes. En contraposición, la mayor dilatación en la liquidación de las operaciones de compras, supone que la empresa PVR debe ofrecer mayor garantía a los proveedores en la liquidación de los créditos concedidos a la empresa. Este efecto se puede analizar de acuerdo con los resultados obtenidos en la Tabla 1 en los ratios de autonomía financiera (RA) obtenido por relación entre el Neto patrimonial y la totalidad del Pasivo exigible de las empresas (Neto/Exigible) y del ratio de garantía obtenido por la relación entre el Activo real de las empresas y sus respectivos Pasivos exigibles (Activo fijo/Exigible).

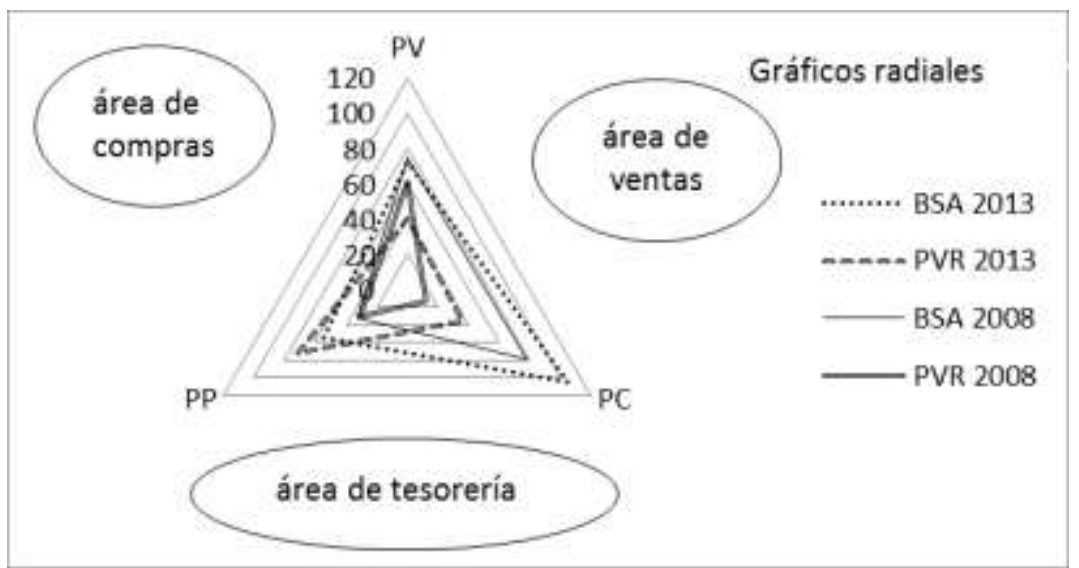

Fig. 1. Gráficos radiales de las empresas Pacífico V Región SA

(PVR) y Belasco SA (BSA) en los años 2008 y 2013.

Tabla 1. Ratios de autonomía financiera y de garantía obtenidos de las empresas BSA y PVR. RAF:

\begin{tabular}{cccccccc} 
Empresa & RATIO $\left.{ }^{*}\right)$ & 2013 & 2012 & 2011 & 2010 & 2009 & 2008 \\
\hline BSA & RAF & 0,859 & 1,256 & 1,139 & 0,847 & 1,451 & 0,838 \\
PVR & RAF & 1,985 & 1,516 & 0,867 & 1,790 & 1,800 & 1,006 \\
& & & & & & & \\
BSA & RG & 1,177 & 1,646 & 1,595 & 1,848 & 1,801 & 1,701 \\
PVR & RG & 2,537 & 2,074 & 1,419 & 2,157 & 2,250 & 1,104 \\
\hline
\end{tabular}

Las empresas BSA y PVR han mantenido su clase gestión durante el periodo analizado. Debido al incremento proporcional de las distancias perimetrales DP1 y DP3 en la empresa BSA, los ratios de la Tabla 1 para esta empresa son más estables que los de la empresa PVR. En contraposición, la alteración de las distancias perimetrales en la empresa PVR no son proporcionales y representan un cambio drástico de gestión. La mayor dilatación de la distancia DP3 respecto de la distancia DP1 en la empresa PVR, en el año 2013, representa un mayor riesgo en la liquidez de las transacciones relacionadas con los proveedores, y este efecto tiene repercusiones en ofrecer mayores garantías al mercado presentando mayores valores que la empresa BSA en los indicadores de la Tabla 1 en el año 2013. Esta actuación se mantiene a lo largo del periodo de estudio y se presenta como una característica general de las dos clases de gestión. Las distancias perimetrales que permiten diferenciar las clases de gestión, son obtenidas a través de la expresión (1) que representa la aplicación del teorema del coseno en cada una de las áreas representadas en un gráfico radial.

$D p_{k}^{2}=p_{i}^{2}+p_{j}^{2}-2 \cos 120 * p_{i} * p_{j}$

$\mathrm{K}=1,2$ y $3 ; 1$, Área de ventas; 2 , Área de tesorería; 3, Área de compras.

$i \neq j ; i=j=v($ ventas), $c$ (cobro), $p$ (pago) 
Las permutas sin repetición de las distancias perimetrales son las distintas clases de gestión que se pueden representar en un gráfico radial de tres dimensiones, se encuentran desarrolladas en la Tabla 2. EI calificativo de positivas o negativas que reciben las clases de gestión en la Tabla 2 se debe a la clase de gestión realizada en el área de tesorería. Cuando la distancia perimetral del área de compras (DP3) es superior a la distancia perimetral del área de ventas (DP1), reciben el calificativo de positivas y cuando la distancia perimetral de ventas (DP1) es superior a la distancia perimetral del área de compras (DP3) es denominada negativa.

Tabla 2: Clases de Gestión financieras en un gráfico radial de tres dimensiones.

\begin{tabular}{|c|c|c|c|}
\hline \multicolumn{2}{|c|}{$\begin{array}{c}\text { Clases de gestión positivas } \\
\text { (DP3 }>\text { DP1) }\end{array}$} & \multicolumn{2}{c|}{$\begin{array}{c}\text { Clases de gestión negativas } \\
\text { (DP1>DP3) }\end{array}$} \\
\hline CLASE A & DP2>DP3>DP1 & CLASE D & DP1>DP3>DP2 \\
\hline CLASE B & DP3>DP2>DP1 & CLASE E & DP1>DP2>DP3 \\
\hline CLASE C & DP3>DP1>DP2 & CLASE F & DP2>DP1>DP3 \\
\hline
\end{tabular}

El análisis de la situación financiera de las empresas a través de indicadores financieros obtenidos en la Tabla 1 es considerado, en esta metodología, como un resultado de la gestión realizada por las entidades. En consecuencia, lo relevante es la clase de gestión adoptada por las compañías para el ejercicio continuado de su actividad. Las clases de gestión adoptadas por las empresas se analizan bajo la hipótesis de que las culturas de las regiones donde están localizadas las empresas son el factor que está presente en las transacciones realizadas y condiciona la clase de gestión adoptada por la compañía. En el ejemplo propuesto en la Fig. 1, las empresas localizas en la región de Las Condes mantienen su clase de gestión en el tiempo y, esta limitación, presupone la existencia factores que evitan adoptar otra clase de gestión de las descritas en la Tabla 2.

\section{LOS MAPAS DE GESTION}

Los mapas de gestión son las distintas clases de gestión adoptadas por las empresas en una región independientemente del sector al que pertenecen. La formación de los mapas de gestión se ha realizado considerando la división administrativa del territorio de Chile. Las empresas elegidas se han obtenido utilizando la base de datos Orbis con licencia de la Universidad de Valencia en España y el criterio utilizado ha sido elegir, como elementos de la muestra, las empresas cotizadas en la bolsa de Chile, obteniendo como resultado 163 empresas. En el estudio se han excluido las entidades del sector financiero, así como aquellas empresas de las que no se ha podido obtener información suficiente para la obtención de los periodos medios de maduración, resultando un total de 127 empresas. El número de empresas evaluadas anualmente se encuentra en la Tabla 3 que contiene el número de clases de gestión adoptadas anualmente. La selección de empresas cotizadas en la composición de la muestra se ha realizado bajo la hipótesis de que aplican criterios de contabilización de las transacciones validados externamente por empresas de auditoria. La primera discriminación realizada sobre la muestra de empresas obtenida ha permitido identificar dos regiones chilenas con significación en la localización de las empresas, Santiago con 79 empresas y Las Condes con 19.

Tabla 3. Clases de gestión de las empresas chilenas.

\begin{tabular}{|l|c|c|c|c|c|c|c|c|c|c|c|}
\hline Global & 2014 & 2013 & 2012 & 2011 & 2010 & 2009 & 2008 & 2007 & 2006 & 2005 & GLOBAL \\
\hline A & 7 & 58 & 57 & 43 & 39 & 32 & 30 & 27 & 25 & 22 & 340 \\
\hline B & 2 & 14 & 10 & 13 & 8 & 11 & 8 & 10 & 6 & 6 & 88 \\
\hline C & 2 & 8 & 8 & 9 & 11 & 10 & 8 & 7 & 13 & 11 & 87 \\
\hline D & 5 & 13 & 13 & 14 & 18 & 16 & 21 & 21 & 19 & 16 & 156 \\
\hline E & 1 & 4 & 6 & 10 & 8 & 9 & 6 & 6 & 6 & 6 & 62 \\
\hline F & 2 & 19 & 21 & 22 & 23 & 22 & 20 & 19 & 16 & 14 & 178 \\
\hline TOTAL & 19 & 116 & 115 & 111 & 107 & 100 & 93 & 90 & 85 & 75 & 911 \\
\hline Positivas & 11 & 80 & 75 & 65 & 58 & 53 & 46 & 44 & 44 & 39 & 515 \\
\hline Negativas & 8 & 36 & 40 & 46 & 49 & 47 & 47 & 46 & 41 & 36 & 396 \\
\hline${ }^{*}$ Santiago* & 2014 & 2013 & 2012 & 2011 & 2010 & 2009 & 2008 & 2007 & 2006 & 2005 & ${ }^{*}$ SANTIAGO* \\
\hline Positivas & 5 & 56 & 54 & 47 & 44 & 39 & 33 & 32 & 29 & 28 & 367 \\
\hline Negativas & 4 & 20 & 21 & 26 & 26 & 25 & 25 & 26 & 25 & 24 & 222 \\
\hline${ }^{*}$ Las Condes & 2014 & 2013 & 2012 & 2011 & 2010 & 2009 & 2008 & 2007 & 2006 & 2005 & *LAS CONDES* \\
\hline Positivas & 5 & 12 & 9 & 9 & 7 & 9 & 6 & 5 & 7 & 4 & 73 \\
\hline Negativas & 3 & 7 & 10 & 9 & 11 & 9 & 10 & 11 & 9 & 4 & 83 \\
\hline
\end{tabular}


Las empresas han ido cambiando de gestión a lo largo del tiempo, como se observa en la Tabla 3. Las consecuencias de adoptar una u otra clase de gestión ya se han analizado anteriormente, en la Fig. 1. Cuando se adoptan clases de gestión positivas las empresas deben ofrecer mayores garantías al mercado que cuando adoptan clases de gestión negativas. El cambio general de gestión se ha producido en la región de Santiago pero no ha tenido el mismo efecto en la región de Las Condes. La prueba del estadístico Chi2 obtenido a través la tabla de contingencia para el conjunto de las empresas de la muestra y las clases de gestión adoptada ha sido 0,4765. En el caso de las clases de gestión producidas en Santiago ha alcanzado el valor de 0,47395 y en el caso de la región de Las Condes el valor de la prueba de Chi2 ha sido 0,9085. El resultado obtenido indica que las clases de gestión adoptadas en la región de Las Condes se han mantenido en el tiempo de acuerdo con la evolución de la actividad económica chilena.

Las empresas chilenas han adoptado preferentemente las clases de gestión positivas frente a las negativas y, entre las clases de gestión positivas, la clase de gestión A han sido la más utilizada desde 2009, cuando la crisis financiera de la Deuda Pública tiene sus inicios. Por otra parte, las clases de gestión de la región de Las Condes presentan un nivel más elevado en las de clase negativa que en las positivas, diferenciándose de la tendencia general de las empresas chilenas. Este factor diferencial ha dirigido el análisis de las clases de gestión en la región del Las Condes que se presenta en un apartado posterior.

Las clases de gestión adoptadas durante el periodo de estudio, ha permitido analizar la economía chilena utilizando la prueba de independencia del indicador Chi2 de cada tabla de contingencia anual de doble entrada, según la distribución de las clases de gestión por regiones (14 regiones) y por sectores económicos (35 sectores). El resultado obtenido en la Tabla 4 indica que existe una mayor relación entre las clases de gestión y la región donde se han generado que la relación entre las clases de gestión y el sector al que pertenecen. Es decir, el factor de localización provoca una mayor alineación de las empresas ante las perturbaciones del mercado que la pertenencia a un sector determinado, adoptando una misma clase de gestión en la región donde desarrollan su actividad, En la Tabla 4 se presentan series temporales de las magnitudes económicas de Chile, obtenidas del Banco Mundial, junto con la evolución de los Chi2 indicados. Las variables macroeconómicas tiene relación con la actividad empresarial y son: NY.GDP.PCAP.KD.ZG: Crecimiento del PIB per cápita (\% anual); SL.IND.EMPL.Z: Empleos en la industria (\% del total de empleos); SL.SRV.EMPL.ZS: Empleos en servicios (\% del total de empleos); FS.LBL.LIQU.GD.ZS: Pasivos líquidos (o M3) como \% del PIB y NY.GDS.TOTL.ZS: Ahorro interno bruto (\% del PIB). Por otra parte, los indicadores Chi2 representan la probabilidad del estadístico por región y por sector e indican el incremento de la dependencia entre las clases de gestión adoptadas y las regiones y sectores industriales. En este sentido, el indicador Chi2 por región muestra una mayor dependencia entre las clases de gestión y la región donde se encuentran localizadas las empresas. No obstante, la tendencia creciente de los indicadores Chi2 tienen su posición más elevada en el año 2010, cuando se produce el efecto de la primera crisis financiera.

Tabla 4. Prueba anual Chi2 por región y por sector

\begin{tabular}{|c|c|c|c|c|c|c|c|}
\hline $\begin{array}{c}\text { Prueba } \\
\text { Anual } \mathrm{CHI} 2\end{array}$ & $\begin{array}{l}\mathrm{CHI} 2\left(^{*}\right) \\
\text { Regional }\end{array}$ & $\begin{array}{l}\mathrm{CHI} 2\left(^{*}\right) \\
\text { Sectorial }\end{array}$ & $\begin{array}{l}\left(^{* *}\right) \text { NY.GDP. } \\
\text { PCAP.KD.ZG }\end{array}$ & $\begin{array}{c}\left({ }^{* *}\right) S L . I N D . \\
\text { EMPL.ZS }\end{array}$ & $\begin{array}{c}\left({ }^{* *}\right) S L . S R V \\
\text { EMPL.ZS }\end{array}$ & $\begin{array}{l}\left({ }^{* *}\right) \text { FS.LBL. } \\
\text { LIQU.GD.ZS }\end{array}$ & $\begin{array}{c}\left({ }^{* *}\right) \text { NY.GDS. } \\
\text { TOTL.ZS }\end{array}$ \\
\hline 2005 & 0,15828 & 0,41613 & 4,356 & 41,800 & 43,500 & 31,372 & 30,095 \\
\hline 2006 & 0,18805 & 0,01053 & 3,230 & 41,700 & 44,300 & 31,623 & 34,035 \\
\hline 2007 & 0,33410 & 0,01650 & 3,992 & 41,900 & 44,600 & 31,887 & 33,092 \\
\hline 2008 & 0,25113 & 0,00413 & 2,155 & 42,300 & 44,700 & 41,413 & 27,931 \\
\hline 2009 & 0,52809 & 0,00703 & $-2,118$ & 41,800 & 45,500 & 39,879 & 27,857 \\
\hline 2010 & 0,59222 & 0,36488 & 4,603 & 23,600 & 65,300 & 38,213 & 28,677 \\
\hline 2011 & 0,80407 & 0,00541 & 4,694 & 23,000 & 66,600 & 42,321 & 26,880 \\
\hline 2012 & 0,97071 & 0,01566 & 4,322 & 23,900 & 65,700 & 45,726 & 25,335 \\
\hline 2013 & 0,89413 & 0,00023 & 3,115 & 23,700 & 67,100 & 49,294 & 23,637 \\
\hline
\end{tabular}

La primera crisis financiera provoca un cambio en la actividad económica de Chile, se incrementa el volumen de empleo en el sector servicios por el del sector industrial. El incremento del PIB per cápita producido en el año 2010 no es consecuente con la política monetaria desarrollada. El incremento de la masa monetaria M3 y la disminución del ahorro monetario provocan que la política monetaria aplicada para superar la primera crisis financiera tenga efectos negativos en el crecimiento posterior del PIB per cápita. Este indicador alcanza niveles próximos al efecto de la primera crisis financiera (Lehman Brothers) en el año 2013, cuando la crisis de la Deuda Soberana en Chile comienza a producir sus efectos, debido a la falta de recursos tributarios para cubrir el gasto presupuestario chileno. La evolución del indicador Chi2 regional de la Tabla 4 muestra el deterioro de la situación económica chilena. El aumento de su valor indica la adopción 
de clases de gestión similares como respuesta común ante las perturbaciones del mercado, limitando las alternativas a adoptar clases de gestión diversificadas. Esta respuesta justifica los resultados obtenidos en la Tabla 3, cuando las empresas adoptan la clase de gestión positiva A y la clase de gestión negativa $\mathrm{F}$ a partir del año 2009.

El resultado del análisis cualitativo, evaluando la actividad económica de Chile, a través del indicado Chi2 en la Tabla 4, indica cómo el indicador Chi2 regional entra en contradicción con los resultados del Chi2 sectorial a partir del año 2009. En ese año, el empleo en el sector servicios aumenta y disminuye en el industrial, y este intercambio continúa hasta el final del periodo de tiempo analizado. Sin embargo, el volumen de clases de gestión financieras se mantiene, indicando un ejercicio continuado de la actividad empresarial. En consecuencia, los resultados de la Tabla 4 indican que los sectores económicos adoptan clases de gestión vinculada a entorno económico donde la empresa ejerce sus actividades. La evolución del indicador Chi2 regional en la Tabla 4 justifica esta proposición e indica que su aumento de valor representa limitaciones a las estrategias de las empresas para adoptar clases de gestión alternativas en el desarrollo de su actividad. El efecto visual del comportamiento advertido se puede analizar en la Fig. 2 del próximo apartado, donde las empresas localizadas cerca del centro de decisiones políticas adoptan clases de gestión positivas y las situadas lejos de centros de influencia adoptan clases de gestión negativas, en ambos casos a lo largo del periodo analizado.

\section{LA DISTRIBUCION GEOGRÁFICA DE LAS CLASES DE GESTION}

La distribución geográfica de las empresas localizadas en las regiones de Santiago (Fig. 2) y de Las Condes (Fig. 3) ha sido elaborada considerando su latitud, longitud y altura utilizando un gráfico de burbujas, donde la mayor o menor amplitud de un círculo representa la mayor o menor altura respecto del nivel del mar. La información de estas variables se ha realizado utilizando la web http://www.coordenadas-gps.com/, y la localización de la capital chilena (Santiago de Chile) ha dado el siguiente resultado: Santiago, Región Metropolitana, Chile. Latitud: -33.44889, Longitud: -70.669265, Altitud: 544 metros. La localización de la Ciudad de Santiago en el mapa geográfico de gestión de la Fig. 2 se encuentra en el centro de área de denominada Santiago 2. Esta área está representada con un círculo de línea paralela y las empresas localizadas en la misma han adoptado las clases de gestión positivas durante todo el periodo analizado. Las clases de gestión positiva están representadas con círculos de línea continua y comparten gestión con tres empresas que utilizan clases de gestión negativas, representadas con círculos de líneas paralelas.

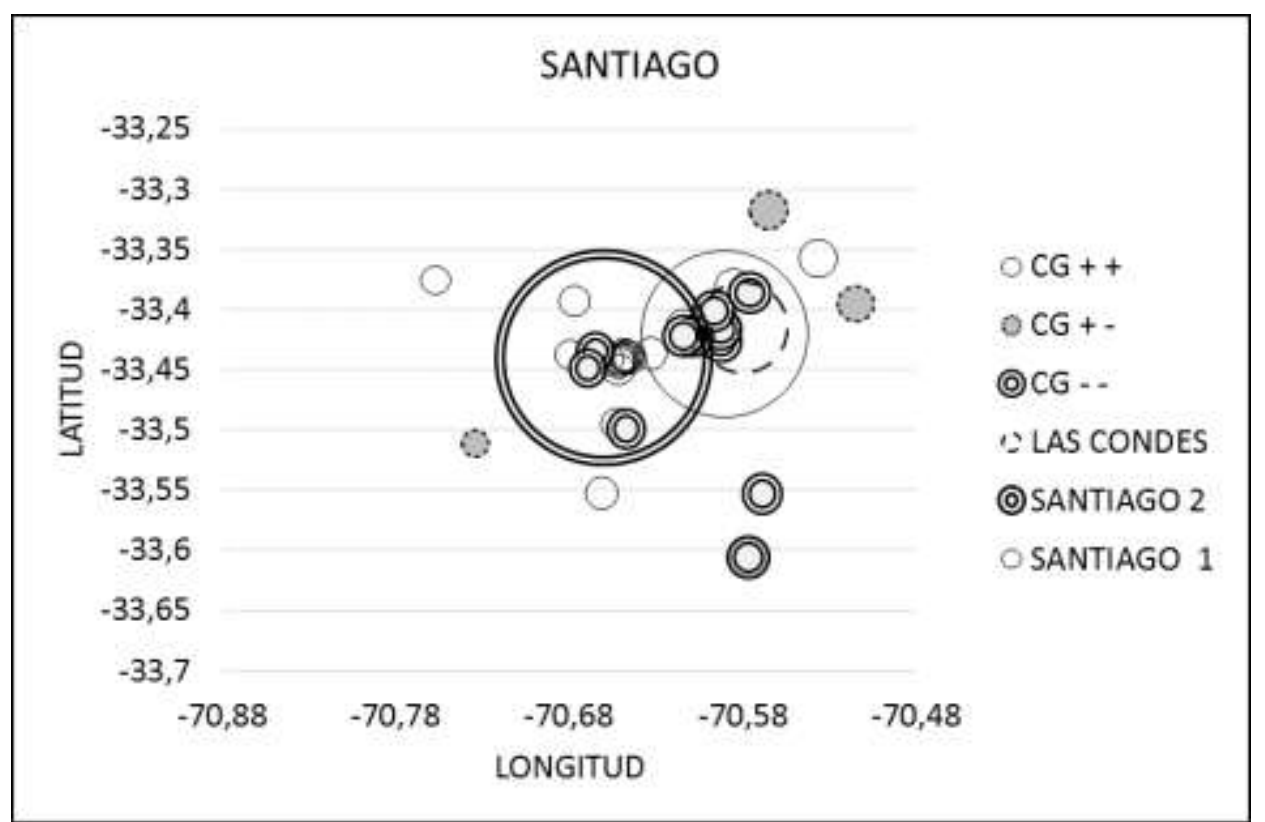

Fig. 2. Mapa geográfico de gestión de la región de Santiago y Las Condes. Variables: $\mathrm{CG}_{++}$: Clases de gestión positivas mantenidas durante todo el periodo. CG+-: Clases de gestión que se transforma de negativas en positivas. CG - -: Clases de gestión negativas que se mantiene durante todo el periodo.

En la región Santiago 1 de la Fig. 2 se encuentra la región de Las Condes, delimitada con trazo discontinuo, y representada más ampliamente en la Fig. 3. Las empresas BSA y PVR, cuyos gráficos radiales de los años 2008 y 2013 han sido representados en la Fig. 1, se encuentran en la Fig. 3. Las coordenadas de latitud, longitud y altura son, respectivamente, para la empresa BSA $-33.416361,-70,603001$ y 640 , y para la 
empresa PVR -33,418918, -70,599273, 640. En esta región las empresas han adoptado las clases de gestión negativas (dirigidas hacia el cliente) como las clases de gestión más adaptadas a la región donde realizan su actividad.

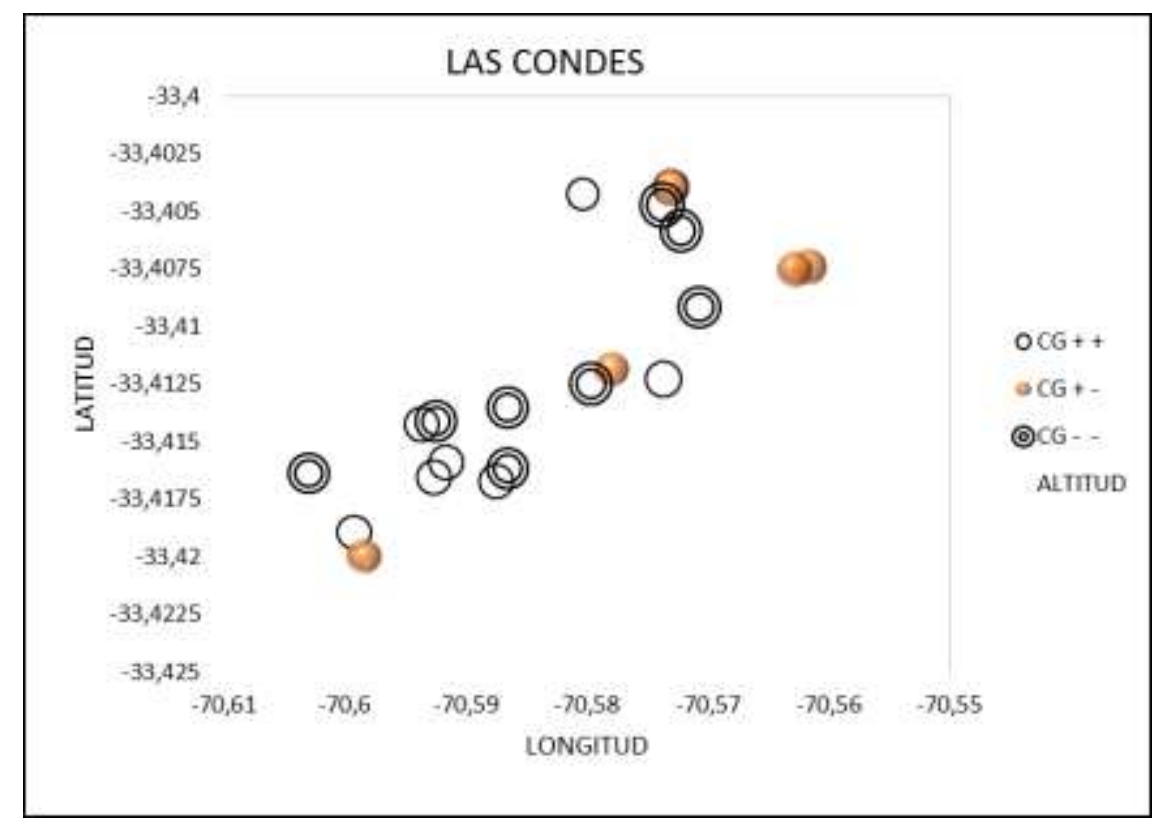

Fig. 3. Clases de gestión en la región de Las Condes.

Esta clase de gestión está localizada en la zona noreste de la región y la clase de gestión basada en la obtención de financiación de los proveedores se localiza, preferentemente, en el cuadrante suroeste de la región. En esta última zona de la región, comparte clases de gestión negativas con los cambios de gestión que se están produciendo también en la zona próxima de Santiago 1 a la región de las Condes, en el mapa Fig. 2. Es decir, independientemente de la clase de sector al que pertenecen las empresas, existe un comportamiento asociado en un momento determinado del tiempo y en un espacio geográfico común. Las empresas con círculos sombreados son las que presentan un cambio de gestión desde las clases negativas a las positivas una vez transcurrido el año 2009, y representan adaptaciones al entorno económico cambiante. En consecuencia, las empresas localizadas en un entorno económico pueden adoptar clases de gestión comunes de acuerdo con las culturas económicas existentes en las regiones donde las empresas desarrollan su actividad. Este factor condiciona la gestión adoptada por las compañías, permite elaborar un espacio socio-económico y favorece dirigir la investigación económica hacia grupos de empresas (agentes económicos) que se identifican bajo una misma clase de gestión.

Por último, la investigación realizada pone de manifiesto que las decisiones macroeconómicas dejan de ser consistentes cuando los entornos económicos de las regiones del país son diferentes y, en consecuencia, el análisis sectorial debe considerar el factor cultural donde las empresas están localizadas y desarrollan sus actividades. El objetivo perseguido en esta investigación, es demostrar que existen culturas económicas donde las empresas desarrollan sus actividades y que, de acuerdo con las teorías existentes, el factor cultural determina o condiciona la clase de gestión adoptada por las empresas en su localización y no entra en conflicto con las teorías sobre la localización de las empresas. Sin embargo, limitaciones como la falta de información sobre las magnitudes económicas de las regiones chilenas o alteraciones en el territorio que modifican las comunicaciones no han sido consideradas por falta de información disponible. Por otra parte, el artículo abre las puertas a otras líneas de investigación sobre la localización de empresas, análisis de empresas y de macroeconomía, utilizando una metodología de investigación a través de indicadores no paramétricos.

\section{CONCLUSIONES}

Con carácter general, las conclusiones alcanzadas se pueden sintetizar en las siguientes: 1) la metodología de los gráficos radiales ha identificado las clases de gestión existentes en una región y justifican las estructuras financieras adoptadas por las empresas, 2) la localización de las empresas en una zona geográfica supone una adaptación a un entorno económico, independientemente del sector al que pertenecen las empresas, 3) las empresas adoptan clases de gestión positiva cuando se encuentran localizadas cenca de los centros de toma decisiones gubernamentales, 4) las empresas han adoptado respuestas comunes ante las crisis financieras, dando lugar a adoptar clases de gestión dirigidas a la 
cartera de proveedores, 5) Las clases de gestión dirigidas al área de compras exigen mayor nivel de garantías que las dirigidas hacia el cliente, 6) La realización de análisis sectoriales entra en contradicción con la localización de las empresas del sector y debe considerar el entorno económico donde las empresas desarrollan su actividad, 7) la clase de gestión es un factor cultural que no entra en contradicción con las teorías existente en el estudio de la localización de las empresas y 8) las decisiones en un área de gestión tiene efectos en el conjunto de las actividades desarrolladas en las empresas que pueden analizarse a través de esta nueva metodología. La interpretación de los sucesos económicos a través de la Geometría inicia un camino diferente para el analista que observa un gráfico radial generado bajo las condiciones indicadas.

\section{REFERENCIAS}

Adams, J. D. y A. B. Jaffe; Bounding the effects of R\&D: An Investigation using matched establishment-firm data. Journal of Economics: 27(4), 700-721 (1996)

Alcacer, J. y W. Chung;. Location strategies for agglomeration economies. Strategic Management Journal: 35(12), 1749-1761, (2014)

Alcacer, J. y M. Delgado; Spatial organization of firms and location choices through the value chain. Management Science (2016)

Allen, T.; Information frictions in trade. Econometrica: 82(6), 2041-2083 (2014)

Anderson, J. E. y E. van Wincoop; Trade costs. Journal of Economic Literature: 42(3), 691-751 (2004)

Ascani, A., R. Crescenzi y S. lammarino, New Economic Geography and Economic Integration: A Review. (en línea: http://www.ub.edu/searchproject/, acceso 8 de abril de 2016), WP1/02 Search Working Paper. Universidad de Barcelona. January (2012)

Bronnenberg, B. J., S. K. Dhar y J.-P. H. Dubé; Brand history, geography, and the persistence of brand shares. Journal of Political Economy: 117(1), 87-115 (2009)

Capó-Vicedo, J., J.V. Tomás-Miquel y M. Expósito-Langa; La gestión del conocimiento en la cadena de suministro: Análisis de la influencia del contexto organizativo. Información tecnológica: 18(1), 127-136, (2007a)

Capó-Vicedo, J., M. Expósito-Langa y J.V. Tomás-Miquel; Creación de redes interorganizativas en un cluster territorial. Información tecnológica: 18(5), 3-10, (2007b)

Cárdenas, U. y J. Ojeda; La nueva economía institucional y la teoría de la implementación. Revista de Economía Institucional: 4(6), 153-169 (2002)

Coval, J. y T. Moskowitz; The geography of investment: Informed trading and asset prices. Journal of Political Economy: 109(4), 811-41 (2001)

Delgado M., M. E. Porter y S. Stern; Clusters and entrepreneurship. Journal of Economic Geography: 10(4), 495-518 (2010)

Du, J., Y. Lu y Z. Tao; Regional institutional strength and FDI location choice in China: Implications for East Asian FDI Source Countries/Areas. Working Paper Series (2008)

Espinosa, F.F y G.E. Salinas; Definición de los requerimientos de información y funciones para la gestión de mantenimiento mediante un proceso de análisis constructivo. doi: 10.4067/S0718-07642015000600009. Información Tecnológica, 26(6), 65-76 (2015)

Espinosa, F.F y G.E. Salinas; Selección de estrategias de mejoramiento de las condiciones de trabajo para la función mantenimiento utilizando la metodología MCDA constructivista. doi: 10.4067/S071807642013000300008. Información Tecnológica, 24(3), 57-72 (2013)

García, A. y E. L. Taboada; Teoría de la empresa: las propuestas de Coase, Alchian y Demsetz, Williamson, Penrose y Nooteboom, (en línea: http://www.izt.uam.mx/economiatyp, acceso 8 de abril), ISSN 0188-3380, Revista Economía: Teoría y práctica, 36, Enero-Junio (2012)

Geyskens, I., J. B. E. Steenkamp y N. Kumar; Make, buy, or ally: A transaction cost theory meta-analysis. Academy of management journal: 49(3), 519-543 (2006) 
Gómez-Guillamón, A. L. D. y M. M. Sanchez-Val. The geographical factor in the determination of audit quality. Revista de Contabilidad: 15(2), 287 (2012)

Hernández, A., C.E. Marulanda y M. López; Análisis de Capacidades de Gestión del Conocimiento para la Competitividad de PYMES en Colombia. doi: 10.4067/S0718-07642014000200013. Información Tecnológica, 25(2), 111-122 (2014)

Hope, O.K., T. Kang, W. Thomas y Y.K. Yoo; Culture and audit choice: a test of the secrecy hypothesis. Journal of Accounting and Public Policy: 27(5), 357-73 (2008)

Huang, R.D. y H. Li; Does the market dole out collective punishment? An empirical analysis of industry, geography, and Arthur Andersen's reputation. Journal of Banking \& Finance: 33(7), 1255-1265 (2009)

Jacobs, J.; Cities and the Wealth of Nations: Principles of Economic Life. Random House, New York (1984)

Kalnins, A. y F. Lafontaine; Multi-unit ownership in franchising: evidence from the fast-food industry in Texas. Journal of Economics: 35 (4), 747-61 (2004)

Kalnins, A. y F. Lafontaine; Too far away? The effect of distance to headquarters on business establishment performance. American Economic Journal: Microeconomics: 5(3), 157-179 (2013)

Krugman, P.; Increasing returns and economic geography. Journal of Political Economy: 99, 483-499 (1991a)

Krugman, P.; Target zones and exchange rate dynamics. Quarterly Journal of Economics: 106, 669-682 (1991b)

Lafontaine, F. y K. L. Shaw; Targeting managerial control: evidence from franchising. Journal of Economics: 36(1), 131-150 (2005)

Lerner, J.; Venture Capitalists and the Oversight of Private Firms. Journal of Finance: 50(1), 301-318 (1995)

Marshall, A.; Principles of Economics; An Introductory Volume. Macmillan and Co., London (1920)

Martínez, L. y Pérez, M.A; Análisis de estrategias de gestión: Aplicaciones polivalentes del gráfico radial. (en línea: http://www.observatorio-iberoamericano.org/RICG/Portal.htm, acceso 8 de abril), ISSN: 1696-294X,. Revista Iberoamericana de Contabilidad de Gestión: 9(17), 1-34 (2011)

Pérez, M.A. The Application of Accounting Methodology of Radar Charts to Analyze the Sector of Sawmilling and Planning of Wood of Austria. doi: 10.14738/abr.34.1254., Archives of Business Research: 3(4) (2015)

Porter, M. E.; What is strategy? Harvard Business Review. November: 61-78 (1996)

Porter, M.E.; The Competitive Advantage of the Nations. The Free Press. Nueva York, USA (1990)

Portes, R. y H. Rey; The determinants of cross-border equity flows. Journal of International Economics: 65(2), 269-296 (2005)

Rauch, J. E.; Business and social networks in international trade. Journal of Economic Literature: 39(4), 1177-1203 (2001)

Ricardo, D.; On the Principles of Political Economy and Taxation. John Murray, Albemarle-Street Eds., London (1817)

Salgado, C.; Teoría de costos de transacción; una breve reseña, (en línea: http://www.redalyc.org/articulo.oa?id=20502604, acceso 8 de abril), ISSN: 0120-3592, Cuadernos de Administración: 16 (26), 61-78. (2003)

Sobrino, J. Localización industrial y concentración geográfica en México; (en línea: http://www.redalyc.org/articulo.oa?id=31244837001, acceso 8 de abril de 2016), ISSN 0186-7210, Estudios Demográficos y Urbanos: 31 (1), 9-56 (2016)

Urbano, D. y, JC., Díaz; Evolución y principios de la Teoría Económica Institucional. Una propuesta de aplicación para el análisis de los factores condicionantes de la creación de empresas. ISSN: 1135-2533, Investigaciones Europeas de Dirección y Economía de la Empresa; 13(3), 183-198 (2007)

Wang, Q., T.J. Wong y L. Xia; State ownership, the institutional environment, and audit choice: evidence from China. Journal of Accounting and Economics: 46(1), 112-134 (2008) 